\title{
UNIQUENESS OF ENTIRE FUNCTIONS AND FIXED POINTS
}

\author{
Jianming Chang AND Mingliang FAng
}

\begin{abstract}
Let $f$ be a nonconstant entire function. If $f, f^{\prime}$ and $f^{\prime \prime}$ have the same fixed points, then $f \equiv f^{\prime}$.
\end{abstract}

\section{Introduction}

Let $f$ be a nonconstant meromorphic function in the whole complex plane. We use the following standard notation of value distribution theory,

$$
T(r, f), m(r, f), N(r, f), \bar{N}(r, f), \ldots
$$

(see Hayman [1], Yang [6]). We denote by $S(r, f)$ any function satisfying

$$
S(r, f)=o\{T(r, f)\},
$$

as $r \rightarrow+\infty$, possibly outside a set of $r$ of finite linear measure.

Let $g$ be a meromorphic function, and let $a, b$ be two complex numbers. If $g(z)=b$ whenever $f(z)=a$, then we denote it by $f(z)=a \Rightarrow g(z)=b$. Thus $f(z)=a \Leftrightarrow g(z)=a$ means $f(z)=a$ if and only if $g(z)=a$.

In 1986, Jank-Muse-Volkmann [3] proved the following result.

THEOREM A. Let $f$ be a nonconstant entire function, and a be a nonzero value. If $f(z)=a \Leftrightarrow f^{\prime}(z)=a$, and $f^{\prime}(z)=a \Rightarrow f^{\prime \prime}(z)=a$, then $f \equiv f^{\prime}$.

In this paper, we extend Theorem A as follows.

THEOREM 1. Let $f$ be a nonconstant entire function, and let $a, c$ be two nonzero constants. If $f(z)=a \Rightarrow f^{\prime}(z)=a, f^{\prime}(z)=a \Rightarrow f^{\prime \prime}(z)=c$, then either $f(z)=A e^{c z / a}+\left(a c-a^{2}\right) / c$ or $f(z)=A e^{c z / a}+a$, where $A$ is a nonzero constant.

2000 Mathematics Subject Classification: 30D35.

Keywords and phrases: Meromorphic function, entire function, uniqueness.

†Supported by the NNSF of China (Grant No. 10071038) and "Qinglan Project" of the Educational Department of Jiangsu Province.

Received February 18, 2002. 
COROLlaRY 2. Let $f$ be a nonconstant entire function, and let a be a nonzero constant. If $f(z)=a \Rightarrow f^{\prime}(z)=a, f^{\prime}(z)=a \Rightarrow f^{\prime \prime}(z)=a$, then either $f \equiv f^{\prime}$ or $f \equiv f^{\prime}+a$.

Remark. In Corollary 2, the case $f \equiv f^{\prime}+a$ occurs.

Let $f(z)=a+A e^{z}$. Then $f^{\prime}(z)=A e^{z}, f^{\prime \prime}(z)=A e^{z}$. Obviously, $f$ satisfies $f(z)=a \Rightarrow f^{\prime}(z)=a, f^{\prime}(z)=a \Rightarrow f^{\prime \prime}(z)=a$, and $f \equiv f^{\prime}+a$.

The main result of this paper is the following

THEOREM 3. Let $f$ be a nonconstant entire function. If $f(z)=z \Leftrightarrow f^{\prime}(z)=$ $z$, and $f^{\prime}(z)=z \Rightarrow f^{\prime \prime}(z)=z$, then $f \equiv f^{\prime}$.

If $f\left(z_{0}\right)=z_{0}$, then $z_{0}$ is called a fixed point of $f$.

COROllary 4. Let $f$ be a nonconstant entire function. If $f, f^{\prime}$ and $f^{\prime \prime}$ have the same fixed points, then $f \equiv f^{\prime}$.

\section{Proof of Theorem 1}

Set

$$
\psi(z)=\frac{c f^{\prime}(z)+a f^{\prime \prime}(z)}{f(z)-a}-\frac{2 c f^{\prime \prime}(z)}{f^{\prime}(z)-a} .
$$

Let $f\left(z_{0}\right)=a$. Then by the assumptions we may suppose that, near $z_{0}$

$$
f(z)=a+a\left(z-z_{0}\right)+\frac{c}{2}\left(z-z_{0}\right)^{2}+b\left(z-z_{0}\right)^{3}+O\left(\left(z-z_{0}\right)^{4}\right),
$$

where $b=f^{(3)}\left(z_{0}\right) / 6$ is a constant. Thus we have

$$
\begin{gathered}
f^{\prime}(z)=a+c\left(z-z_{0}\right)+3 b\left(z-z_{0}\right)^{2}+O\left(\left(z-z_{0}\right)^{3}\right), \\
f^{\prime \prime}(z)=c+6 b\left(z-z_{0}\right)+O\left(\left(z-z_{0}\right)^{2}\right) .
\end{gathered}
$$

Hence

$$
\begin{gathered}
\frac{c f^{\prime}(z)+a f^{\prime \prime}(z)}{f(z)-a}=\frac{2 c}{z-z_{0}}+6 b+O\left(z-z_{0}\right), \\
\frac{2 c f^{\prime \prime}(z)}{f^{\prime}(z)-a}=\frac{2 c}{z-z_{0}}+6 b+O\left(z-z_{0}\right) .
\end{gathered}
$$

Thus we obtain

$$
\psi\left(z_{0}\right)=0 .
$$

Next we consider two cases. 
CASE 1. $f^{\prime \prime} / f^{\prime} \equiv c / a$, that is $c f^{\prime} \equiv a f^{\prime \prime}$. Then we get

$$
f^{\prime}(z)=\frac{c A}{a} e^{(c / a) z}, \quad f(z)=A e^{(c / a) z}+B,
$$

where $A \neq 0, B$ are two constants. obtain

If there exists $z_{0}$ satisfying $f\left(z_{0}\right)=a$, then by the assumptions and (2.5) we

$$
f(z)=A e^{(c / a) z}+\frac{a c-a^{2}}{c} .
$$

If there doesn't exist $z_{0}$ satisfying $f\left(z_{0}\right)=a$, then by (2.5) we get

$$
f(z)=a+A e^{(c / a) z},
$$

where $A$ is a nonzero constant.

CASE 2. $f^{\prime \prime} / f^{\prime} \not \equiv c / a$. Then by the assumptions we have

$$
\begin{aligned}
N\left(r, \frac{1}{f^{\prime}-a}\right) & \leq N\left(r, \frac{1}{f^{\prime \prime} / f^{\prime}-c / a}\right) \leq T\left(r, \frac{f^{\prime \prime}}{f^{\prime}}\right)+O(1) \\
& =N\left(r, \frac{f^{\prime \prime}}{f^{\prime}}\right)+S(r, f)=\bar{N}\left(r, \frac{1}{f^{\prime}}\right)+S(r, f) .
\end{aligned}
$$

In the following, we consider two subcases.

CASE 2.1. $\quad \psi \neq \equiv 0$. Then by $(2.1)$ and (2.4) we get

$$
\begin{aligned}
N\left(r, \frac{1}{f-a}\right) & \leq N\left(r, \frac{1}{\psi}\right) \leq T(r, \psi)+O(1) \\
& \leq N_{0}\left(r, \frac{1}{f^{\prime}-a}\right)+S(r, f),
\end{aligned}
$$

where $N_{0}\left(r, 1 /\left(f^{\prime}-a\right)\right)$ is the counting function for those zero points of $f^{\prime}(z)-a$ which are not zero points of $f(z)-a$.

Thus by the assumption and (2.7) we obtain

$$
2 N\left(r, \frac{1}{f-a}\right) \leq N\left(r, \frac{1}{f^{\prime}-a}\right)+S(r, f) .
$$

On the other hand, by Nevanlinna first fundamental theorem we have

$$
\begin{aligned}
m\left(r, \frac{1}{f-a}\right) & \leq m\left(r, \frac{1}{f^{\prime}}\right)+S(r, f) \\
& \leq T\left(r, f^{\prime}\right)-N\left(r, \frac{1}{f^{\prime}}\right)+S(r, f) \\
& \leq T(r, f)-N\left(r, \frac{1}{f^{\prime}}\right)+S(r, f)
\end{aligned}
$$




$$
\begin{aligned}
& =T\left(r, \frac{1}{f-a}\right)-N\left(r, \frac{1}{f^{\prime}}\right)+S(r, f) \\
& =m\left(r, \frac{1}{f-a}\right)+N\left(r, \frac{1}{f-a}\right)-N\left(r, \frac{1}{f^{\prime}}\right)+S(r, f) .
\end{aligned}
$$

Thus

$$
N\left(r, \frac{1}{f^{\prime}}\right) \leq N\left(r, \frac{1}{f-a}\right)+S(r, f) .
$$

Hence by (2.6), (2.8) and (2.9) we get

$$
N\left(r, \frac{1}{f-a}\right)=N\left(r, \frac{1}{f^{\prime}-a}\right)+S(r, f)=S(r, f) .
$$

By Milloux's inequality (see $[1,6]$ )

$$
T(r, f) \leq N\left(r, \frac{1}{f-a}\right)+N\left(r, \frac{1}{f^{\prime}-a}\right)+S(r, f) .
$$

Thus by (2.10) and (2.11) we get $T(r, f)=S(r, f)$, a contradiction.

CASE 2.2. $\psi \equiv 0 . \quad$ That is

$$
\frac{c f^{\prime}(z)+a f^{\prime \prime}(z)}{f(z)-a} \equiv \frac{2 c f^{\prime \prime}(z)}{f^{\prime}(z)-a} .
$$

Thus by $f(z)=a \Rightarrow f^{\prime}(z)=a$ and (2.12) we deduce that $f(z)=a \Leftrightarrow f^{\prime}(z)=a$. Hence, $f^{\prime \prime}(z)=0 \Rightarrow f^{\prime}(z)=0$.

Set

$$
\phi(z)=\frac{a f^{\prime \prime}(z)-c f^{\prime}(z)}{f(z)-a} .
$$

Since $f^{\prime \prime} / f^{\prime} \not \equiv c / a$, we get $\phi \not \equiv 0$.

Let $f^{\prime}\left(z_{0}\right)=0$ and $f^{\prime \prime}\left(z_{0}\right) \neq 0$. Then by (2.12) we get

$$
f\left(z_{0}\right)=\frac{2 a c-a^{2}}{2 c} .
$$

Differentiating the two sides of (2.12) we get

$$
\begin{aligned}
& \frac{\left[c f^{\prime \prime}(z)+a f^{\prime \prime \prime}(z)\right][f(z)-a]-f^{\prime}(z)\left[c f^{\prime}(z)+a f^{\prime \prime}(z)\right]}{[f(z)-a]^{2}} \\
& \equiv \frac{2 c f^{\prime \prime \prime}(z)\left[f^{\prime}(z)-a\right]-2 c\left[f^{\prime \prime}(z)\right]^{2}}{\left[f^{\prime}(z)-a\right]^{2}} .
\end{aligned}
$$

Thus by (2.14), (2.15), $f^{\prime}\left(z_{0}\right)=0$ and $f^{\prime \prime}\left(z_{0}\right) \neq 0$, we obtain

$$
f^{\prime \prime}\left(z_{0}\right)=c \text {. }
$$


Hence we have

$$
\phi\left(z_{0}\right)=-\frac{2 c^{2}}{a} .
$$

Next we divide two subcases.

CASE 2.2.1. $\phi(z) \not \equiv-2 c^{2} / a$. Then by (2.17),

$$
\bar{N}\left(r, \frac{1}{f^{\prime}}\right)-\bar{N}\left(r, \frac{1}{f^{\prime \prime}}\right) \leq \bar{N}\left(r, \frac{1}{\phi+2 c^{2} / a}\right) \leq T(r, \phi)+S(r, f) .
$$

Obviously, by (2.13), Logarithmic Derivative Lemma (see $[1,6]$ ) and the assumptions we get

$$
T(r, \phi)=S(r, f) .
$$

Thus we get

$$
\bar{N}\left(r, \frac{1}{f^{\prime}}\right)-\bar{N}\left(r, \frac{1}{f^{\prime \prime}}\right)=S(r, f) .
$$

By $f^{\prime \prime}(z)=0 \Rightarrow f^{\prime}(z)=0,(2.13)$ and (2.19) we have

$$
\bar{N}\left(r, \frac{1}{f^{\prime \prime}}\right) \leq \bar{N}\left(r, \frac{1}{\phi}\right)=T(r, \phi)+O(1)=S(r, f) .
$$

Hence

$$
\bar{N}\left(r, \frac{1}{f^{\prime}}\right)=S(r, f) .
$$

Thus by Milloux's inequality, (2.22) and (2.6), we get $T(r, f)=S(r, f)$, a contradiction.

CASE 2.2.2. $\phi \equiv-2 c^{2} / a$. That is

$$
a f^{\prime \prime}(z)-c f^{\prime}(z)+\frac{2 c^{2}}{a}[f(z)-a]=0,
$$

for $z \in \mathbf{C}$.

If there exists $z_{0}$ such that $f^{\prime \prime}\left(z_{0}\right)=0$, then by $f^{\prime \prime}(z)=0 \Rightarrow f^{\prime}(z)=0$ and (2.23) we get $f^{\prime}\left(z_{0}\right)=0$ and $f\left(z_{0}\right)=a$, which contradicts $f(z)=a \Rightarrow f^{\prime}(z)=a$. Hence, $f^{\prime \prime}(z) \neq 0$.

Solving the equation (2.23) we obtain

$$
f(z)=c_{1} e^{\lambda_{1} z}+c_{2} e^{\lambda_{2} z}+a,
$$

where $\lambda_{1}$ and $\lambda_{2}$ are solutions of the equation $a z^{2}-c z+2 c^{2} / a=0$, and $c_{1}, c_{2}$ are two constants.

Thus

$$
f^{\prime \prime}(z)=c_{1} \lambda_{1}^{2} e^{\lambda_{1} z}+c_{2} \lambda_{2}^{2} e^{\lambda_{2} z}
$$


Since $f^{\prime \prime}(z) \neq 0$, we deduce from (2.25) that either $c_{1}=0$ or $c_{2}=0$. Without loss of generality, we assume that $c_{2}=0$, then

$$
f(z)=c_{1} e^{\lambda_{1} z}+a .
$$

Thus by $f^{\prime}(z)=a \Rightarrow f^{\prime \prime}(z)=c$ and (2.26) we get

$$
f(z)=A e^{(c / a) z}+a .
$$

The proof of Theorem 1 is complete.

\section{Proof of Theorem 3}

Firstly, we consider the case that $f$ is a transcendental entire function.

Obviously, we have

$$
\begin{aligned}
m(r, & \left.\frac{1}{f-z}\right)+m\left(r, \frac{1}{f^{\prime}-z}\right) \\
& \leq m\left(r, \frac{1}{f^{\prime \prime}}\right)+m\left(r, \frac{1}{f^{\prime \prime}-1}\right)+S(r, f) \\
& \leq m\left(r, \frac{1}{f^{\prime \prime}}+\frac{1}{f^{\prime \prime}-1}\right)+S(r, f) \\
& \leq m\left(r, \frac{1}{f^{\prime \prime \prime}}\right) \leq T\left(r, f^{\prime \prime \prime}\right)+S(r, f) \\
& \leq T\left(r, f^{\prime}\right)+S(r, f) .
\end{aligned}
$$

Hence by Nevanlinna's first fundamental theorem, we have

$$
\begin{aligned}
& T(r, f-z)+T\left(r, f^{\prime}-z\right) \\
& \quad \leq N\left(r, \frac{1}{f-z}\right)+N\left(r, \frac{1}{f^{\prime}-z}\right)+T\left(r, f^{\prime}\right)+S(r, f) .
\end{aligned}
$$

By $f(z)=z \Leftrightarrow f^{\prime}(z)=z$, and $f^{\prime}(z)=z \Rightarrow f^{\prime \prime}(z)=z$, it is easy to see that

$$
N\left(r, \frac{1}{f^{\prime}-z}\right)=N\left(r, \frac{1}{f-z}\right)+S(r, f) .
$$

Thus by (3.1) and (3.2) we have

$$
T(r, f) \leq 2 N\left(r, \frac{1}{f-z}\right)+S(r, f) .
$$

Set

$$
H(z)=\frac{f^{\prime \prime}(z)}{f^{\prime}(z)-1}-\frac{z}{z-1}
$$


If $H \equiv 0$, then by (3.4) we get

$$
\frac{f^{\prime \prime}(z)}{f^{\prime}(z)-1} \equiv \frac{z}{z-1} \text {. }
$$

Thus we have

$$
\begin{gathered}
f^{\prime}(z)=1+C(z-1) e^{z} \\
f(z)=z+C(z-2) e^{z}+A .
\end{gathered}
$$

where $A, C(\neq 0)$ are two constants.

Hence by (3.5), (3.6) and $f(z)=z \Leftrightarrow f^{\prime}(z)=z$, we know that $f^{\prime}(z)=z$ have the unique solution $z_{0}=2-A$ with $z_{0} \neq 0,1$. But it is clear that $f^{\prime}(z)=z$ have infinitely many solutions, a contradiction. Hence $H \not \equiv 0$, that is

$$
\frac{f^{\prime \prime}(z)}{f^{\prime}(z)-1} \not \equiv \frac{z}{z-1} .
$$

Thus by the assumption of the theorem, we have

$$
\begin{aligned}
N\left(r, \frac{1}{f-z}\right) & \leq N\left(r, \frac{1}{H}\right)+O(\log r) \\
& \leq T(r, H)+S(r, f) \\
& \leq N\left(r, \frac{f^{\prime \prime}}{f^{\prime}-1}\right)+S(r, f) \\
& \leq \bar{N}\left(r, \frac{1}{f^{\prime}-1}\right)+S(r, f) .
\end{aligned}
$$

Hence by (3.7) and (3.3) we get

$$
T(r, f) \leq 2 \bar{N}\left(r, \frac{1}{f^{\prime}-1}\right)+S(r, f) .
$$

Set

$$
\begin{gathered}
\phi(z)=\frac{f^{\prime}(z)-1}{f(z)-z}-\frac{f^{\prime \prime}(z)-1}{f^{\prime}(z)-z}, \\
\psi(z)=\frac{(z-1) f^{\prime \prime}(z)-z\left[f^{\prime}(z)-1\right]}{f(z)-z} .
\end{gathered}
$$

Obviously, by Logarithmic Derivative Lemma (see $[1,6]$ )

$$
m(r, \phi)=S(r, f), \quad m(r, \psi)=S(r, f) .
$$

Let $z_{0}$ satisfy $f\left(z_{0}\right)=z_{0}$ and $z_{0} \neq 0,1$. Then by assumption we may assume that, near $z_{0}$ 


$$
f(z)=z_{0}+z_{0}\left(z-z_{0}\right)+\frac{z_{0}}{2}\left(z-z_{0}\right)^{2}+\frac{f^{\prime \prime \prime}\left(z_{0}\right)}{6}\left(z-z_{0}\right)^{3}+\cdots,
$$

Thus we have

$$
\begin{gathered}
f^{\prime}(z)=z_{0}+z_{0}\left(z-z_{0}\right)+\frac{f^{\prime \prime \prime}\left(z_{0}\right)}{2}\left(z-z_{0}\right)^{2}+\cdots, \\
f^{\prime \prime}(z)=z_{0}+f^{\prime \prime \prime}\left(z_{0}\right)\left(z-z_{0}\right)+\cdots .
\end{gathered}
$$

By (3.12)-(3.14) we get

$$
N(r, \phi)=S(r, f), \quad N(r, \psi)=S(r, f) .
$$

Thus we have

$$
T(r, \phi)=S(r, f), \quad T(r, \psi)=S(r, f) .
$$

By (3.12)-(3.14) and (3.9)-(3.10) we get

$$
\phi\left(z_{0}\right)=\frac{f^{\prime \prime \prime}\left(z_{0}\right)-z_{0}}{2\left(1-z_{0}\right)},
$$

and

$$
\psi\left(z_{0}\right)=f^{\prime \prime \prime}\left(z_{0}\right)-z_{0}-1
$$

Thus we obtain

$$
2\left(z_{0}-1\right) \phi\left(z_{0}\right)+\psi\left(z_{0}\right)+1=0 .
$$

If $2(z-1) \phi(z)+\psi(z)+1 \not \equiv 0$, then by $(3.16)$

$$
\begin{aligned}
N\left(r, \frac{1}{f-z}\right) & \leq N\left(r, \frac{1}{2(z-1) \phi+\psi+1}\right)+O(\log r) \\
& \leq T(r, \phi)+T(r, \psi)+S(r, f) \leq S(r, f) .
\end{aligned}
$$

Thus by (3.3) and (3.17) we get a contradiction: $T(r, f)=S(r, f)$. Hence

$$
2(z-1) \phi(z)+\psi(z)+1 \equiv 0 .
$$

Now let $z_{1}$ satisfy $f^{\prime}\left(z_{1}\right)=1$ and $z_{1} \neq 1$. Then by $f(z)=z \Leftrightarrow f^{\prime}(z)=z$, we know that $f\left(z_{1}\right) \neq z_{1}$. Thus by (3.12) and (3.13) we have

$$
\begin{gathered}
\phi\left(z_{1}\right)=\frac{f^{\prime \prime}\left(z_{1}\right)-1}{z_{1}-1}, \\
\psi\left(z_{1}\right)=\frac{\left(z_{1}-1\right) f^{\prime \prime}\left(z_{1}\right)}{f\left(z_{1}\right)-z_{1}} .
\end{gathered}
$$

Hence by (3.18)-(3.20) we obtain

$$
\left[2 f\left(z_{1}\right)-z_{1}-1\right] f^{\prime \prime}\left(z_{1}\right)=f\left(z_{1}\right)-z_{1} .
$$


If $2 f\left(z_{1}\right)-z_{1}-1=0$, then $f\left(z_{1}\right)=z_{1}$, a contradiction. Hence $2 f\left(z_{1}\right)-$ $z_{1}-1 \neq 0$. Thus

$$
f^{\prime \prime}\left(z_{1}\right)=\frac{f\left(z_{1}\right)-z_{1}}{2 f\left(z_{1}\right)-z_{1}-1} .
$$

Therefore by (3.19)-(3.21) we get

$$
\begin{aligned}
& \phi\left(z_{1}\right)=\frac{1-f\left(z_{1}\right)}{\left(z_{1}-1\right)\left[2 f\left(z_{1}\right)-z_{1}-1\right]}, \\
& \psi\left(z_{1}\right)=\frac{z_{1}-1}{2 f\left(z_{1}\right)-z_{1}-1} .
\end{aligned}
$$

By (3.9), (3.10) and (3.21) we get

$$
\phi^{\prime}\left(z_{1}\right)=\frac{f^{\prime \prime \prime}\left(z_{1}\right)}{z_{1}-1}+\frac{1}{2 f\left(z_{1}\right)-z_{1}-1}+\frac{\left[f\left(z_{1}\right)-1\right]^{2}}{\left(z_{1}-1\right)^{2}\left[2 f\left(z_{1}\right)-z_{1}-1\right]^{2}},
$$

and

$$
\psi^{\prime}\left(z_{1}\right)=\frac{\left(z_{1}-1\right) f^{\prime \prime \prime}\left(z_{1}\right)}{f\left(z_{1}\right)-z_{1}}-\frac{z_{1}-1}{2 f\left(z_{1}\right)-z_{1}-1} .
$$

By (3.18) we get

$$
2 \phi(z)+2(z-1) \phi^{\prime}(z)+\psi^{\prime}(z) \equiv 0 .
$$

Thus we have

$$
2 \phi\left(z_{1}\right)+2\left(z_{1}-1\right) \phi^{\prime}\left(z_{1}\right)+\psi^{\prime}\left(z_{1}\right)=0 .
$$

By (3.22)-(3.24) and (3.27) we get

$$
\frac{2\left[1-f\left(z_{1}\right)\right]}{\left(z_{1}-1\right)^{2}} \psi\left(z_{1}\right)+2 f^{\prime \prime \prime}\left(z_{1}\right)+2 \psi\left(z_{1}\right)+\frac{2\left[f\left(z_{1}\right)-1\right]^{2}}{\left(z_{1}-1\right)^{3}} \psi^{2}\left(z_{1}\right)+\psi^{\prime}\left(z_{1}\right)=0 .
$$

By (3.25) we get

$$
f^{\prime \prime \prime}\left(z_{1}\right)=\frac{\left[\psi^{\prime}\left(z_{1}\right)+\psi\left(z_{1}\right)\right]\left[f\left(z_{1}\right)-z_{1}\right]}{z_{1}-1} .
$$

Thus by (3.28) and (3.29) we have

$$
\begin{aligned}
& \frac{2\left[1-f\left(z_{1}\right)\right]}{\left(z_{1}-1\right)^{2}} \psi\left(z_{1}\right)+\frac{2\left[\psi^{\prime}\left(z_{1}\right)+\psi\left(z_{1}\right)\right]\left[f\left(z_{1}\right)-z_{1}\right]}{z_{1}-1} \\
& +2 \psi\left(z_{1}\right)+\frac{2\left[f\left(z_{1}\right)-1\right]^{2}}{\left(z_{1}-1\right)^{3}} \psi^{2}\left(z_{1}\right)+\psi^{\prime}\left(z_{1}\right)=0 .
\end{aligned}
$$

By (3.23) we get 


$$
f\left(z_{1}\right)=\frac{z_{1}+1}{2}+\frac{z_{1}-1}{2 \psi\left(z_{1}\right)} .
$$

Hence by (3.30) and (3.31), we have

$$
2\left(z_{1}-1\right) \psi^{\prime}\left(z_{1}\right)+\psi^{3}\left(z_{1}\right)+2\left(z_{1}-1\right) \psi^{2}\left(z_{1}\right)+\left(2 z_{1}-3\right) \psi\left(z_{1}\right)=0 .
$$

Let

$$
\Delta=2(z-1) \psi^{\prime}(z)+\psi^{3}(z)+2(z-1) \psi^{2}(z)+(2 z-3) \psi(z) .
$$

If $\Delta \not \equiv 0$, then

$$
\bar{N}\left(r, \frac{1}{f^{\prime}-1}\right) \leq N\left(r, \frac{1}{\Delta}\right)+O(\log r) \leq T(r, \Delta)+S(r, f) \leq S(r, f) .
$$

Thus by (3.8) and (3.33) we get a contradiction: $T(r, f)=S(r, f)$.

Hence, $\Delta \equiv 0$, that is

$$
2(z-1) \psi^{\prime}(z)+\psi^{3}(z)+2(z-1) \psi^{2}(z)+(2 z-3) \psi(z) \equiv 0 .
$$

Obviously, by (3.34), $\psi$ is an entire function. We claim that $\psi$ is not transcendental. Indeed, if $\psi$ is transcendental, then by (3.34) we have

$$
\begin{aligned}
3 T(r, \psi) & =3 m(r, \psi)=m\left(r, \psi^{3}\right) \\
& \left.=m\left(r, 2(z-1) \psi^{2}+(2 z-3) \psi+2(z-1) \psi^{\prime}\right)\right) \\
& \leq m(r, \psi)+m\left(r, 2(z-1) \psi+(2 z-3)+2(z-1) \frac{\psi^{\prime}}{\psi}\right) \\
& \leq 2 m(r, \psi)+S(r, \psi)=2 T(r, \psi)+S(r, \psi) .
\end{aligned}
$$

Thus we get a contradiction: $T(r, \psi)=S(r, \psi)$. Hence $\psi$ is a polynomial. Next, by simple computation, we deduce that either $\psi \equiv 0$ or $\psi \equiv-1$.

If $\psi \equiv 0$, Then by (3.10) we get

$$
(z-1) f^{\prime \prime}(z) \equiv z\left[f^{\prime}(z)-1\right],
$$

which means $H \equiv 0$, a contradiction.

If $\psi \equiv-1$, then by (3.18) we know $\phi \equiv 0$. Thus by (3.9) we have

$$
\frac{f^{\prime}(z)-1}{f(z)-z} \equiv \frac{f^{\prime \prime}(z)-1}{f^{\prime}(z)-z}
$$

Next we can easily deduce that $f \equiv f^{\prime}$.

Now we prove that $f$ can not be a polynomial.

By simple computation, $f$ can not be a polynomial with $\operatorname{deg} f \leq 2$. Next we prove that $f$ can not be a polynomial with $\operatorname{deg} f \geq 3$. Suppose that there exists such polynomial $f$ with $f(z)=z \Leftrightarrow f^{\prime}(z)=z$ and $f^{\prime}(z)=z \Rightarrow f^{\prime \prime}(z)=z$, and $d=\operatorname{deg} f \geq 3$. Let $z_{1}, z_{2}, \ldots, z_{n}$ be the fixed points of $f$. Then we have 


$$
\begin{aligned}
& f(z)=z+A\left(z-z_{1}\right)^{\alpha_{1}}\left(z-z_{2}\right)^{\alpha_{2}} \cdots\left(z-z_{n}\right)^{\alpha_{n}}, \\
& f^{\prime}(z)=z+B\left(z-z_{1}\right)^{\beta_{1}}\left(z-z_{2}\right)^{\beta_{2}} \cdots\left(z-z_{n}\right)^{\beta_{n}},
\end{aligned}
$$

and

$$
f^{\prime \prime}(z)=z+C\left(z-z_{1}\right)^{\gamma_{1}}\left(z-z_{2}\right)^{\gamma_{2}} \cdots\left(z-z_{n}\right)^{\gamma_{n}} p(z),
$$

where $p(\not \equiv 0)$ is a polynomial, and $A, B, C$ are three non-zero constants and $\left\{\alpha_{j}\right\},\left\{\beta_{j}\right\},\left\{\gamma_{j}\right\}(j=1,2, \ldots, n)$ are positive integers satisfying

$$
\sum_{j=1}^{n} \alpha_{j}=d, \quad \sum_{j=1}^{n} \beta_{j}=d-1, \quad \sum_{j=1}^{n} \gamma_{j}+\operatorname{deg} p=d-2 .
$$

From (3.35) and (3.36) we obtain

$$
1+A \sum_{i=1}^{n} \alpha_{i}\left(z-z_{i}\right)^{\alpha_{i}-1} \prod_{j \neq i}\left(z-z_{j}\right)^{\alpha_{j}} \equiv z+B \prod_{j=1}^{n}\left(z-z_{j}\right)^{\beta_{j}} .
$$

If $\alpha_{j} \geq 2$, then by (3.39) we get $z_{j}=1$. Similarly, we know that if $\beta_{j} \geq 2$, then $z_{j}=1$. Without loss of generality, we assume that $j=1$. Thus by (3.35)(3.36) and (3.38) we have

$$
\begin{gathered}
f(z)=z+A(z-1)^{\alpha_{1}}\left(z-z_{2}\right) \cdots\left(z-z_{n}\right), \\
f^{\prime}(z)=z+B(z-1)^{\alpha_{1}-1}\left(z-z_{2}\right) \cdots\left(z-z_{n}\right) .
\end{gathered}
$$

If $\alpha_{1} \geq 3$, then by (3.40) we get $f(1)=1$ and $f^{\prime \prime}(1)=0$, which contradicts $f(z)=z \Rightarrow f^{\prime \prime}(z)=z$. Thus $\alpha_{1}=2$. Hence by (3.37)-(3.38), and (3.41) we have

$$
\begin{aligned}
& f^{\prime}(z)=z+B(z-1)\left(z-z_{2}\right) \cdots\left(z-z_{n}\right) . \\
& f^{\prime \prime}(z)=z+C(z-1)\left(z-z_{2}\right) \cdots\left(z-z_{n}\right) .
\end{aligned}
$$

Thus by (3.42) and (3.43) we get a contradiction: $\operatorname{deg} f^{\prime}=\operatorname{deg} f^{\prime \prime}$. The proof of Theorem 3 is complete.

\section{REFERENCES}

[1] W. K. Hayman, Meromorphic Functions, Oxford Math. Monogr., Clarendon Press, Oxford, 1964.

[2] G. G. Gundersen, Meromorphic functions that share finite values with their derivative, J. Math. Anal. Appl., 75 (1980), 441-446.

[ 3 ] G. Jank, E. Mues and L. Volkmann, Meromorphe Funktionen, die mit ihrer ersten und zweiten Ableitung einen endlichen Wert teilen, Complex Variables Theory Appl., 6 (1986), 51-71.

[4] E. Mues and N. Steinmetz, Meromorphe Funktionen, die mit ihrer Ableitung Werte teilen, Manuscripta Math., 29 (1979), 195-206.

[5] L. A. Rubel AND C.-C. YANG, Values shared by an entire function and its derivative, Complex Analysis (J. D. Buckholtz and T. J. Suffridge eds.), Lecture Notes in Math. 599, Springer-Verlag, Berlin, 1977, 101-103. 
[6] L. YANG, Value Distribution Theory, Springer-Verlag, Berlin, 1993.

[7] H. X. YI AND C. C. YANG, Unicity Theory of Meromorphic Functions, Science Press, Beijing, 1995.

Department of Mathematics

Changshu College

Changshu, Jiangsu 215500

P. R. China

e-mail: jmwchang@pub.sz.jsinfo.net

Department of Mathematics

NANJING Normal UNIVERSITY

NANJING 210097

P. R. CHINA

e-mail: mlfang@pine.njnu.edu.cn 